\title{
IMMERSIONS AND EMBEDDINGS OF PROJECTIVE SPACES
}

\author{
S. FEDER AND D. M. SEGAL
}

\begin{abstract}
The topic is embedding and immersions of complex and quaternionic projective spaces. The results are obtained using spin representations and relating these with the various $K$-theories in which they occur. A numerical result on nonembeddings and and nonimmersions is obtained.
\end{abstract}

The purpose of this note is to revise and complete the results of [1] and [2]. The reader is referred to the aforementioned articles for a statement of the problem, notation and techniques. We recall only that $\ddagger$ and $\varnothing$ stand for "does not immerse" and "does not embed" respectively; $C \boldsymbol{P}_{n}$ denotes the complex and $H P_{n}$ the quaternion projective space and $K G$ denotes the theory of stable $G$-bundles.

The complete statement of results would be as follows:

THEOREM 1. $C P_{n} \nsubseteq R^{4 n-2 \alpha(n)-1}$ for all $n$.

THEOREM 2. $C P_{n} \not R^{4 n-2 \alpha(n)}$ for all $n$.

THEOREM 3. $H P_{n} \not R^{8 n-2 \alpha(n)-2} ; H P_{n} \not R^{8 n-2 \alpha(n)}$ if $\alpha(n) \equiv 0(4)^{1} ; H P_{n}$ 必 $R^{8 n-2 \alpha(n)-3} ; H P_{n} \nsubseteq R^{8 n-2 \alpha(n)-2}$ if $\alpha(n) \not \equiv 1$ (4) and $H P_{n} \ddagger R^{8 n-2 \alpha(n)-1}$ if $\alpha(n) \equiv 0$ or $3(4)$.

$\alpha(n)$ is the number of l's in the dyadic expansion of $n$.

All the results are obtained using $K$-theory, namely the spin representation. The improvements come from the knowledge that in various dimensions the complex spin representations are restrictions of quaternionic ones or come from real representations.

Theorem 1 has been proven for $n$ odd. To remove this restriction we consider $\mu=H+v$ where $\nu$ is the normal bundle of $C P_{n} \subseteq R^{2 n+2 k+1}$ (the result is better than that obtained considering $\left.C P_{n-1} \subset C P_{n}\right)$. Now $\mu$ is a spin bundle. We let $x=H+\bar{H}-2, x$ is the generator of the subalgebra of

Received by the editors April 22, 1971.

AMS 1970 subject classifications. Primary 57A35, 57D40; Secondary 55B15.

Key n'ords and phrases. Embedding, immersion, spin representation, $K$-theory, complex projective space, quaternionic projective space.

1 We thank the referee for pointing out that this result of $\mathrm{K}$. Mayer also follows from our techniques. 
selfconjugate elements in $K U\left(C P_{n}\right)$ and $x^{n / 2+1}=0$. We have $\mu=2 n+2 k+$ $3-n H$ and

$$
\lambda_{t}\left(\mu_{u}\right)=(1+t)^{2 n+2 k+3}(1+t H)^{-n}(1+t \bar{H})^{-n} .
$$

Using the fact that $H \bar{H}=1$ we find $\lambda_{t}\left(\mu_{u}\right)=(1+t)^{2 k+3}\left(1+t x /(1+t)^{2}\right)^{-n}$ and $\lambda_{1}\left(\mu_{n}\right)=2^{2 k+3}(1+x / 4)^{-n}$. Therefore $\Delta(\mu)=2^{k+1}(1+x / 4)^{-n / 2}$ is an element of $K U\left(C P_{n}\right)$. Checking the coefficient of $x^{n / 2}$ we get $\pm 2^{k+1}\left(\begin{array}{c}n-1 \\ n / 2\end{array}\right) 2^{-n}$. This must be an integer and since the highest power of 2 dividing $\left(\begin{array}{c}a+b \\ a\end{array}\right)$ is $\alpha(a)+\alpha(b)-\alpha(a+b)$ we must have

$$
k+1-n+\alpha(n / 2)+\alpha(n / 2-1)-\alpha(n-1) \geqq 0 .
$$

For $n$ even $\alpha(n / 2)=\alpha(n)$ and $\alpha(n / 2-1)=\alpha(n-1)$ so the last inequality is $k \geqq n-\alpha(n)$.

A similar argument removes the restriction $n \equiv 1$ (2) in Theorem 2; note, however, that the proof as given in [2] proves the nonembedding result and not nonimmersion as was claimed. This is because we assumed that $\Delta^{+}-\Delta^{-}=0$ which is the case for embeddings but not for immersions in general.

The statements in Theorem 3 which give no restriction on $\alpha(n)$ were obtained in [2]. To prove the other part of the statement we need the following results from the theory of group representations (e.g. [3, p. 193]):

$$
\Delta(v) \in \operatorname{Im}\left(K O\left(H P_{n}\right) \rightarrow K U\left(H P_{n}\right)\right)
$$

whenever $\operatorname{dim} \nu \equiv 0,1,2,6$ or $7 \bmod 8$;

$$
\Delta^{+}(v) \in \operatorname{Im}\left(K O\left(H P_{n}\right) \rightarrow K U\left(H P_{n}\right)\right)
$$

whenever $\operatorname{dim} \nu \equiv 0 \bmod 8$;

$$
\Delta(v) \in \operatorname{Im}\left(K S p\left(H P_{n}\right) \rightarrow K U\left(H P_{n}\right)\right)
$$

whenever $\operatorname{dim} v \equiv 2,3,4,5$ or $6 \bmod 8$;

$$
\Delta^{+}(v) \in \operatorname{Im}\left(K \operatorname{Sp}\left(H P_{n}\right) \rightarrow K U\left(H P_{n}\right)\right)
$$

whenever $\operatorname{dim} v \equiv 4 \bmod 8$.

To prove that $H P_{n} \nsubseteq R^{8 n-2 \alpha(n)-2}$ for $\alpha(n) \not \equiv 1$ (4) let us assume that such an immersion were possible; the normal bundle $\nu$ would be a $4 n-2 \alpha(n)-2$ dimensional spin-bundle and we would have $\Delta(v) \in K U\left(H P_{n}\right)$ given by

$$
\Delta(v)=2^{2 n-\alpha(n)-1}(1+z / 4)^{-(n+1)}(1+z / 2) .
$$

Since the image $K O\left(H P_{n}\right) \rightarrow K U\left(H P_{n}\right)$ is generated by $2 z$ and $z^{2}\left(z^{n+1}=0\right)$ the result follows for $n$ even. Indeed, $4 n-2 \alpha(n)-2 \equiv 0,2$ or $6 \bmod 8$ provided $\alpha(n) \not \equiv 1$ (4) and the coefficient of $z^{n-1}$ in $\Delta(v)$ is (e.g. [2])

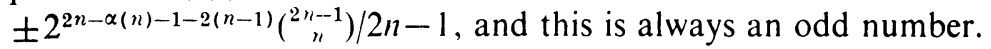


For $n$ odd we observe that $4 n-2 \alpha(n)-2 \equiv 2,4$ or $6 \bmod 8$ if $\alpha(n) \not \equiv 1$ (4) and so

$$
\Delta(v) \in \operatorname{Im}\left(K S p\left(H P_{n}\right) \rightarrow K U\left(H P_{n}\right)\right) .
$$

This image is only a submodule and is generated by $z^{2 i+1}$ and $2 z^{2 i}$. Now $n-1$ is even and the same calculation yields the result.

If $\alpha(n) \equiv 0$ or 3 (4) the above argument gives a contradiction to $H P_{n} \subseteq R^{8 n-2 \alpha(n)-1}$.

The nonembedding result $H P_{n} \not R^{8 n-2 \alpha(n)}$ provided $\alpha(n) \equiv 0$ (4) is obtained by the above argument for $\Delta^{+}(v)=\frac{1}{2} \Delta(v)$.

\section{REFERENCES}

1. S. Feder, Immersions and embeddings in complex projective spaces, Topology 4 (1965), 143-158. MR 32 \#1717.

2. - Non-immersion theorems for complex and quaternionic projective spaces, Bol. Soc. Mat. Mexicana (2) 11 (1966), 62-67. MR 38 \#721.

3. D. Husemoller, Fiber bundles, McGraw-Hill, New York, 1966. MR 37 \#4821.

Department of Mathematics, University of Illinois, Chicago, Illinois 60680

Department of Mathematics, University of Washington, Seattle, Washington 98195

Department of Mathematics, City College, City University of New York, NEW YORK, NEW YORK 10031 